\title{
Ethereum's Recursive Length Prefix in ACL2
}

\author{
Alessandro Coglio \\ Kestrel Institute \\ http://www. kestrel.edu
}

\begin{abstract}
Recursive Length Prefix (RLP) is used to encode a wide variety of data in Ethereum, including transactions. The work described in this paper provides a formal specification of RLP encoding and a verified implementation of RLP decoding, developed in the ACL2 theorem prover. This work has led to improvements to the Ethereum documentation and additions to the Ethereum test suite.
\end{abstract}

\section{Problem and Contribution}

Errors in cryptocurrency code may lead to particularly direct financial losses. This applies not only to smart contracts, but also to the underlying execution engines, to wallets, and to other critical components.

In Ethereum [20], Recursive Length Prefix (RLP) [21, Page 'RLP'] [23, Appendix B] is used to encode a wide variety of data, including transactions. It is thus important for this fundamental building block to be specified precisely and implemented correctly.

The work described in this paper contributes to this goal by providing a formal specification of RLP encoding and a verified implementation of RLP decoding, developed in the ACL2 theorem prover [11]. The development is available [18, Path books/kestrel/ethereum/rlp] and thoroughly documented [17, Topic $r l p]$. Some excerpts of the development shown in this paper are slightly simplified for brevity.

This work has led to improvements to the Ethereum Yellow Paper [23] and to the Ethereum Wiki [21], which are major components of the Ethereum documentation. It has also led to additions to the Ethereum test suite [19], which contains tests for all Ethereum implementations. See Section 5 for details.

This work is part of an ongoing effort to develop, in ACL2, a formal specification and a verified implementation of a complete Ethereum client [12]. This formal specification will also be useful for formally verifying existing client implementations, smart contracts at the level of the EVM (Ethereum Virtual Machine), and the compilation of higher-level programming languages to EVM code.

\section{Background}

Ethereum uses RLP to encode transactions, which may include smart contract code to run on the EVM. It also uses RLP to encode blocks, whose hashing plays a critical role in the blockchain paradigm. Thus, errors in RLP encoders and decoders may lead, among other problems, to unexpected smart contract code, or to incorrect block verification or mining.

RLP is specified informally in the Ethereum wiki ('WK' for short) [21, Page 'RLP'] and more formally in the Ethereum Yellow Paper ('YP' for short) [23, Appendix B].

RLP encodes nested sequences of bytes into flat sequences of bytes that can be decoded back into the original nested sequences. These nested sequences are finitely branching ordered trees with flat sequences of bytes at their leaf nodes and no additional information at the branching nodes. An example is $\langle\langle[1,2,3],\langle\rangle\rangle,[255],[]\rangle$, where $[\ldots]$ denote leaf nodes and $\langle\ldots\rangle$ denote branching nodes; this tree is

G. Passmore, R. Gamboa (Eds.): ACL2 Workshop 2020 EPTCS 327, 2020, pp. 108-124 doi $10.4204 / E P T C S .327 .11$
(C) Alessandro Coglio

This work is licensed under the Creative Commons Attribution License. 

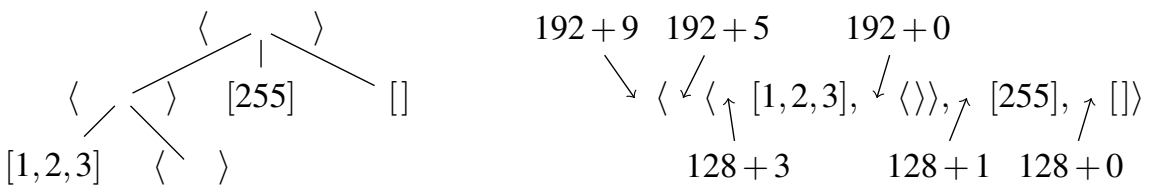

Figure 1: An example RLP tree and its encoding.

depicted in Figure 1](left). The leaf tree [] with the empty sequence of bytes differs from the branching tree \langle\rangle with no subtrees ${ }^{1}$

WK calls the trees 'items', the branching trees 'lists', and the leaf trees 'strings' or 'byte arrays'; it uses Python notations "..." for strings (with the implicit assumption that characters consist of 8 bits) and $[\ldots, \ldots]$ for lists; it also uses lists $[\ldots, \ldots]$ of numbers and characters for flat sequences of bytes. YP uses a more explicit tree terminology; it uses a mathematical notation $(\ldots, \ldots)$ for both leaf and branching nodes, as well as for flat sequences of bytes. This paper uses the notations $[\ldots]$ and $\langle\ldots\rangle$ shown earlier and in Figure 1.

RLP prescribes to encode non-negative integers by first representing them as base-256 big-endian byte arrays without leading zeros (not even a 0 byte for the integer 0 , which is represented as the empty byte array), and then encoding those as any other leaf trees. Other than that, RLP does not prescribe encodings for any data types, delegating that to the "users" of RLP.

Leaf and branching trees are encoded into byte arrays by recursively adding a few extra bytes before most nodes to indicate the kind of node (leaf or branching) and the length of the subsequent bytes. These extra bytes tell the decoder how to reconstruct the nodes of the tree.

Leaf trees $[. .$.$] are encoded by encoding their byte arrays as follows. Singleton byte arrays whose$ only byte is below 128 are encoded as themselves, without extra bytes. Other byte arrays whose length is in the range $0-55$ have an extra starting byte in the range 128-183, which is 128 plus the length. Longer byte arrays have from 1 to 8 extra bytes that contain the base-256 big-endian no-leading-zeros length, preceded by an extra byte in the range 184-191 that indicates the number of the base-256 big-endian bytes-184 for 1,185 for $2, \ldots$, and 191 for 8 .

Branching trees $\langle\ldots\rangle$ are encoded by first recursively encoding the subtrees and concatenating all the encodings, and then adding one or more extra bytes to indicate the length of the concatenated encodings: if the length is in the range 0-55, there is a single extra byte in the range 192-247, which is 192 plus the length; otherwise, there are from 1 to 8 extra bytes that contain the base-256 big-endian no-leadingzero length, preceded by an extra byte in the range 248-255 that indicates the number of the base-256 big-endian bytes-248 for 1,249 for $2, \ldots$, and 255 for 8 .

For instance, $\langle\langle[1,2,3],\langle\rangle\rangle,[255],[]\rangle$ is encoded as $[201,197,131,1,2,3,192,129,255,128]$. This is illustrated in Figure 1 (right).

There is a symmetry between leaf and branch encodings with respect to small and large lengths: the first 56 values of the 64 values in the range 128-191 or 192-255 are used for small lengths from 0 to 55 , while the remaining 8 values are used for large lengths up to $2^{64}-1$, i.e. the maximum value representable in 8 digits in base 256 , since $256^{8}=2^{64}$. However, leaf trees have an additional shorter encoding, when they consist of single bytes below 128 .

\footnotetext{
${ }^{1}$ In this paper, a 'leaf tree' is one that consists of just one leaf node (which is also necessarily the root), while a 'branching tree' is one that has (at least) a branching node as root.
} 


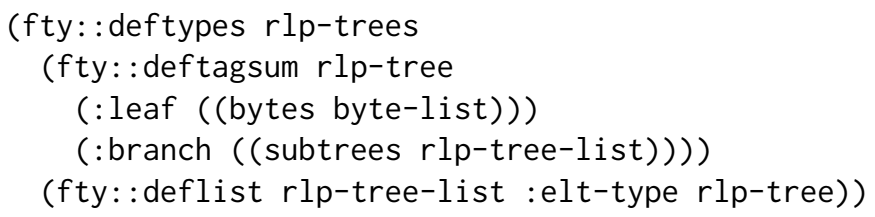

Figure 2: Formalization of RLP trees in ACL2.

\section{RLP Encoding}

\subsection{Tree Structures}

As explained in Section 2, RLP encodes trees (i.e. nested byte sequences) into flat byte sequences (i.e. byte arrays). These trees are formalized in Figure 2, using the FTY library [17, Topic fty].

The fty: : deftagsum defines a tagged sum type (disjoint union) called rlp-tree. Leaf trees are tagged by :leaf; branching trees are tagged by :branch. A leaf tree has a single component called bytes of type byte-list, which consists of lists of natural numbers below 256 (its definition is not shown). A branching tree has a single component called subtrees of type rlp-tree-list, defined by the fty: :deflist as consisting of lists of trees; :elt-type specifies the element type of the lists. The surrounding fty: : deftypes introduces $r l p$-tree and $r l p$-tree-list as mutually recursive types, with rlp-trees as the name of the ensemble.

In more detail, the fty: :deftagsum introduces a recognizer rlp-treep, a fixer rlp-tree-fix, constructors $r l p$-tree-leaf and $r l p$-tree-branch for leaf and branching trees, accessors $r l p-t r e e-$ leaf->bytes and rlp-tree-branch->subtrees for leaf and branching trees, and several theorems about these functions, which are all guard-verified. The fty: :deflist introduces a recognizer rlptree-listp, a fixer $r l p-t r e e-l i s t-f i x$, and theorems about these functions and existing list functions; no specific constructors or accessors are introduced by fty: : deflist, since the generic ones for lists can be used.

In essence, Figure 2 defines the set $\mathbb{T}$ of trees as a least fixpoint of the recursive set equation $\mathbb{T}=$ $\{0, \ldots, 255\}^{\star} \uplus \mathbb{T}^{\star}$, where $\{0, \ldots, 255\}$ is the set of bytes, $X^{\star}$ is the set of all the finite sequences of elements in $X$ (Kleene star), and $X \uplus Y$ is the disjoint union of $X$ and $Y$. This is consistent with the set-theoretic notation in YP, which uses $\mathbb{O}$ for $\{0, \ldots, 255\}, \mathbb{B}$ for $\mathbb{O}^{\star}$, and $\mathbb{L}$ for $\mathbb{T}^{\star}$.

\subsection{Encoding Functions}

The encoding of RLP trees into byte arrays is formalized in Figure 3

The function rlp-encode-bytes formalizes the encoding of the byte arrays at the leaves of RLP trees, using the define enhancement of defun [17, Topic define], which supports type annotations for arguments (abbreviating guards) and results (abbreviating theorems). The function rlp-encode-bytes takes as input a list of bytes and returns as output a pair [17, Topic mv] consisting of a boolean error flag and a list of bytes that is the RLP encoding of the argument. The error flag is $t$ exactly when the input consists of $2^{64}$ or more bytes: in this case, the input cannot be RLP-encoded, and the second component of the output is just nil, but irrelevant. Otherwise, the error flag is nil and the second component of the output is the encoding.

First, rlp-encode-bytes fixes the argument to be a list of bytes via the fixer byte-list-fix (a no-op under the guard), using the $b *$ enhancement of let* [17, Topic $b *]$. Then there are four cases: 


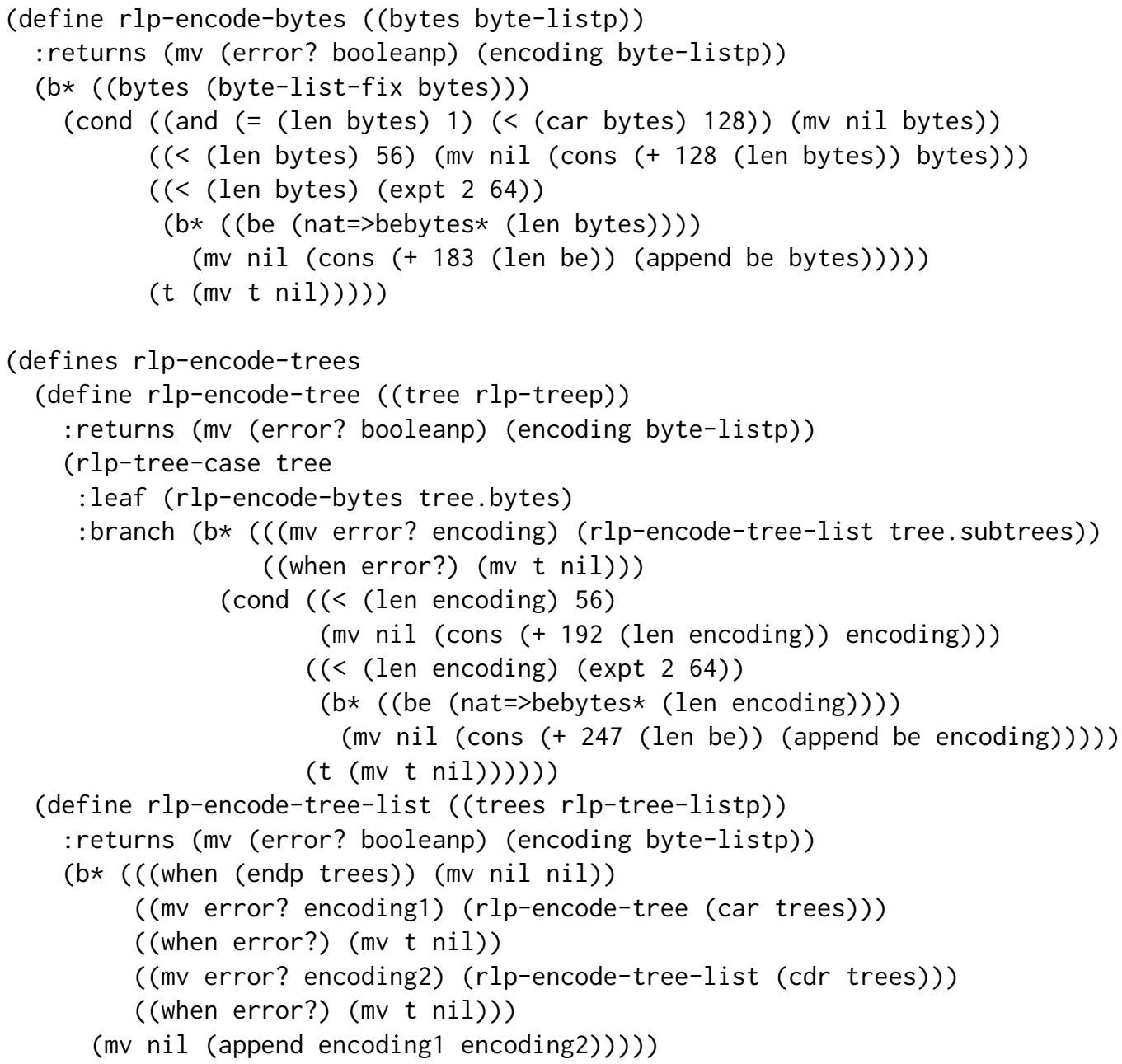

Figure 3: Formalization of RLP encoding in ACL2.

1. If the list consists of one byte and that byte is below 128, the operation is successful (i.e. the error? result is nil) and the encoding is the singleton list of the byte itself.

2. Otherwise, if the list consists of $l<56$ bytes, the operation is successful and the encoding is obtained by prepending the byte $128+l$ to the list of bytes.

3. Otherwise, if the list consists of $l<2^{64}$ bytes, the operation is successful and the encoding is obtained by prepending (i) the byte $183+l l$ to the concatenation of (ii) the base-256 big-endian no-leading-zeros representation of $l$ of length $l l$ ('length of length') and (iii) the initial list of bytes. The library function nat=>bebytes* turns a natural number into a list of bytes as big-endian digits in base 256, without leading zeros.

4. Otherwise, the operation fails: the list of bytes is too long to be encoded. To encode a list of $2^{64}$ or more bytes, 9 or more base-256 digits would be needed, i.e. it would be $l l \geq 9$, and therefore the first byte would be 192 or more, overlapping with the encoding of branching trees and preventing a decoder from discriminating leaf and branching trees by the first byte of an encoding.

The function $r l p$-encode-bytes formalizes the function $R_{\mathrm{b}}$ in YP, which returns one result: either the encoding (a byte sequence), or $\varnothing$ if the input byte sequence cannot be encoded. In the ACL2 for- 
mulation, it is more convenient to return two results, so that each result always has the same type. The function nat=>bebytes*, mentioned above, formalizes the function BE in YP.

The function rlp-encode-tree formalizes the encoding of (both branching and leaf) trees. It takes as input a tree and returns as output a pair consisting of a boolean error flag and a list of bytes that is the RLP encoding of the argument- the same output types as rlp-encode-bytes.

First, rlp-encode-tree performs a case analysis on the argument via the macro rlp-tree-case, generated by the fty: : deftagsum in Figure 2. If tree is a leaf tree, rlp-encode-bytes is called on the list of bytes in the leaf; the variable tree.bytes is bound to ( $r l p$-tree-leaf->bytes tree) by rlp-tree-case. If instead tree is a branching tree, $r l p$-encode-tree-list is called to encode each subtree and concatenate their encodings (more details below); the variable tree. subtrees is bound to ( $r l p$-tree-branch->subtrees tree) by $r l p$-tree-case. The two results of $r l p$-encode-tree-list are simultaneously bound to error? and encoding via b*'s support for binding patterns such as (mv ...). If $r l p$-encode-tree-list returns an error, rlp-encode-tree returns an error too, via $b *$ 's early-exit construct ( (when ...) ...): if any subtree cannot be encoded, the tree cannot be encoded either. Otherwise, there are three cases:

1. If the concatenated subtree encodings consist of $l<56$ bytes, the overall operation is successful and the overall encoding is obtained by prepending the byte $192+l$ to the concatenated subtree encodings.

2. Otherwise, if the concatenated subtree encodings consist of $l<2^{64}$ bytes, the overall operation is successful and the overall encoding is obtained by prepending (i) the byte $247+l l$ to the concatenation of (ii) the base-256 big-endian no-leading-zeros representation of $l$ of length $l l$ and (iii) the concatenated subtree encodings.

3. Otherwise, the overall operation fails, because the tree is too large to be encoded. To encode a tree whose concatenated subtree encodings consist of $2^{64}$ or more bytes, 9 or more base- 256 digits would be needed, i.e. it would be $l l \geq 9$, and therefore the first byte would be 256 or more, which would not actually be a byte.

The function rlp-encode-tree-list formalizes the encoding of a list of (sub)trees and the concatenation of the resulting encodings. It takes as input a list of trees and returns as output a pair consisting of a boolean error flag and a list of bytes that are the concatenated encodings of the argument trees; these are the same output types as rlp-encode-tree. When the list of trees is empty, the result is the empty list of bytes nil. Otherwise, the first tree is encoded, the remaining list of trees is encoded, and the two resulting lists of bytes are concatenated. If any tree in the list cannot be encoded, an error is returned.

The functions $r l p$-encode-tree and $r l p$-encode-tree-list are mutually recursive. The macro defines [17, Topic defines] groups mutually recursive functions that are introduced via define (and also provides some enhancements over mutual-recursion), with rlp-encode-trees as the name of the ensemble. Termination is proved automatically, based on the decreasing size of the argument trees, which is explicitly supplied as measure (not shown).

The function rlp-encode-tree formalizes the function RLP in YP; the :branch case of the definition of $r l p$-encode-tree formalizes the function $R_{1}$ in YP. The function $r l p$-encode-tree-list formalizes the function $s$ in YP.

The functions $r l p$-encode-... are guard-verified; their guard verification proofs are essentially automatic, with just a hint (not shown) to locally enable a rewrite rule that may be somewhat expensive to be always enabled. The proofs of the result type theorems of the rlp-encode-... functions are also proved essentially automatically, with just a hint (not shown) to locally enable a definition that is normally kept disabled and to locally enable a linear arithmetic rule that may be somewhat expensive to be always enabled. All these proofs make use of existing library rules about the functions called by the 


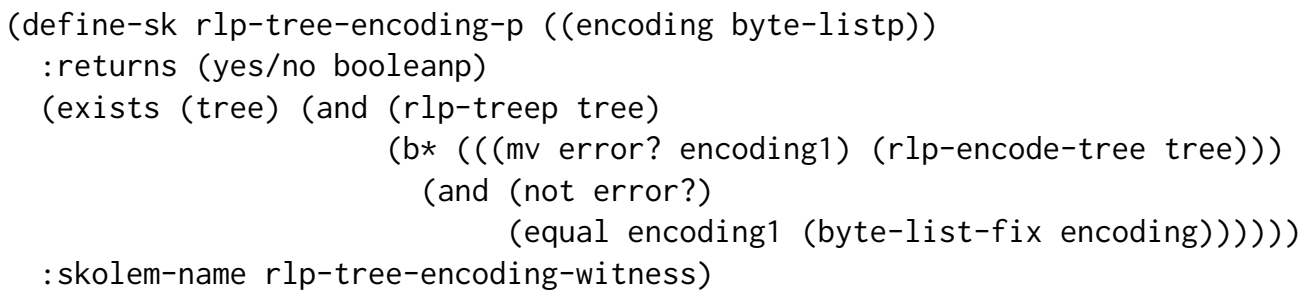

Figure 4: Formalization of valid RLP encodings in ACL2.

rlp-encode- ... functions.

The rlp-encode- . . . functions provide a high-level specification of RLP encoding that also happens to be executable. The definitions of these functions correspond very closely to the definitions in YP. These definitions are also similar to the Python reference code in WK.

\subsection{Valid Encodings}

Valid encodings are formalized in Figure 4, using the define-sk enhancement [17, Topic define-sk] of defun-sk [17, Topic defun-sk], which provides conveniences similar to define.

The predicate $r l p$-tree-encoding- $p$ returns $t$ exactly on the byte arrays that encode some trees. Roughly speaking, this predicate characterizes the image of $r l p$-encode-tree, restricted to the second result of that function, and subject to the constraint that the first result is nil. This predicate has a declarative, non-executable definition.

\subsection{Decodability Properties}

RLP encodings are decodable, i.e. trees can be recovered from their encodings - a basic requirement for any encoding method. This is expressed by the theorems in Figure 5; (i) the RLP encoding function is injective, i.e. no two distinct trees have the same encoding; and (ii) the RLP encoding function is prefixunambiguous, i.e. no valid tree encoding is a strict prefix of another one. The second property ensures the ability to decode from byte streams without "end-of-encoding" markers: if a valid encoding could be a strict prefix of another valid encoding, then after reading the former, a decoder could either stop there or proceed to decode a longer encoding, giving rise to an ambiguity.

Typically, the injectivity of a function $f$ is stated as $[x \neq y \Longrightarrow f(x) \neq f(y)]$, with $x$ and $y$ universally quantified, or equivalently as $[f(x)=f(y) \Longrightarrow x=y]$. Because $[x=y \Longrightarrow f(x)=f(y)]$ is always trivially true, injectivity can be equivalently stated as $[f(x)=f(y) \Longleftrightarrow x=y]$, which is usable as a rewrite rule. If $f$ operates on values of a type (predicate) $\tau$, its injectivity restricted to values of that type can be stated as $[\tau(x) \wedge \tau(y) \Longrightarrow(f(x)=f(y) \Longleftrightarrow x=y)]$; if $f$ implicitly or explicitly fixes values outside $\tau$ via a fixer $\phi$ for $\tau$, injectivity can be equivalently stated as $[f(x)=f(y) \Longleftrightarrow \phi(x)=\phi(y)] \mathrm{n}^{2}$ which is generally preferable as a rewrite rule because it has no hypotheses. This is the formulation in Figure 5, applied to the encoding results and with the necessary hypotheses that the error? results are nil; the results are obtained via mv-nth [17, Topic mv-nth].

First, a theorem rlp-encode-bytes-injective, not shown here but analogous to rlp-encodetree-injective (with rlp-encode-tree and $r l p$-tree-fix replaced with $r l p$-encode-bytes and

\footnotetext{
${ }^{2}$ This is obtained by replacing $x$ and $y$ with $\phi(x)$ and $\phi(y)$ in $[\tau(x) \wedge \tau(y) \Longrightarrow(f(x)=f(y) \Longleftrightarrow x=y)]$, and using the fact that $\forall z . \tau(\phi(z))$ (i.e. $\phi$ is a fixer for $\tau$ ) and $\forall z . f(\phi(z))=f(z)$ (i.e. $f$ fixes its argument). Conversely, assuming $\tau(x)$ and $\tau(y)$, $[f(x)=f(y) \Longleftrightarrow \phi(x)=\phi(y)]$ reduces to $[f(x)=f(y) \Longleftrightarrow x=y]$ because $\forall z . \tau(z) \Longrightarrow \phi(z)=z$ (i.e. $\phi$ is identity over $\tau$ ).
} 


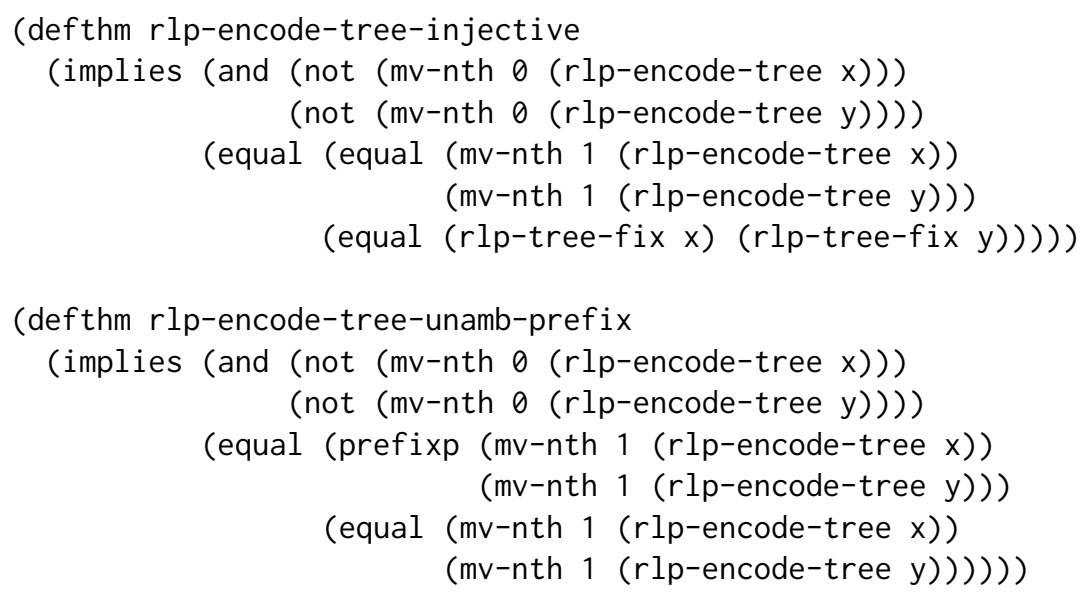

Figure 5: ACL2 theorems asserting the decodability of RLP encodings.

byte-list-fix), is proved by first proving a variant lemma (not shown) with byte-listp hypotheses on $\mathrm{x}$ and $\mathrm{y}$ and without byte-list-fix (i.e. the form $[\tau(x) \wedge \tau(y) \Longrightarrow(f(x)=f(y) \Longleftrightarrow x=y)]$ above). The lemma is proved automatically, once the definition of rlp-encode-bytes is enabled via a hint, which leads ACL2 to consider nine cases - three for $\mathrm{x}$ and three for $\mathrm{y}$, corresponding to the three branches in the definition of $r l p$-encode-bytes; see the documentation [17, Topic $r l p]$ for details. Then the theorem is easily proved from the lemma via a hint (not shown) to use the instance of the lemma where $x$ and $y$ are replaced with (byte-list-fix $x$ ) and (byte-list-fix y). Attempting to prove the theorem directly, with just the hint to enable the definition of $r l p$-encode-bytes, fails.

Then, the theorem rlp-encode-tree-injective is also proved by first proving a variant lemma analogous to the one for $r l p$-encode-bytes-injective described above (i.e. the form $[\tau(x) \wedge \tau(y) \Longrightarrow$ $(f(x)=f(y) \Longleftrightarrow x=y)]$ above). Since $r l p$-encode-tree is mutually recursive with $r l p-e n c o d e-$ tree-list, this lemma is proved by induction along with a similar lemma about the injectivity of rlp-encode-tree-list (not shown). The induction schemas that ACL2 automatically generates for trees and for the encoding functions, which operate on single variables, do not work for these lemmas: a new induction schema (not shown) is provided that operates on two variables simultaneously, such as $\mathrm{x}$ and $\mathrm{y}$ in the lemmas; this new induction schema is more general than its use in this proof. Some of the base and step cases of the induction are proved automatically, while others make use of some fairly specific lemmas (not shown); see the documentation [17, Topic rlp] for details. The base case, in which $x$ or $y$ is a leaf tree, makes use of $r l p$-encode-bytes-injective, the injectivity theorem for rlp-encode-bytes.

The injectivity of the RLP encoding functions could be alternatively proved by defining RLP decoding functions, proving that the latter are left inverses of the former, and using the inverse property to prove injectivity ${ }^{3}$ In contrast, the injectivity proofs explained above, which are not particularly difficult, are solely in terms of the encoding functions, which is more elegant and abstract in my opinion.

The prefix-unambiguity theorem in Figure 5 says that if a valid encoding is a prefix of another valid encoding, then the two encodings are equal; similarly to the injectivity theorem, this theorem also states the obvious converse implication, making the theorem a more useful rewrite rule. The library function prefixp tests whether the first argument is a (not necessarily strict) prefix of the second argument. Thus,

\footnotetext{
${ }^{3}$ If $f$ has a left inverse $g$, then given $f(x)=f(y)$, and applying $g$ to both sides to obtain $g(f(x))=g(f(y))$, the left inverse property yields $g(f(x))=x=y=g(f(y))$. Thus $f$ is injective.
} 


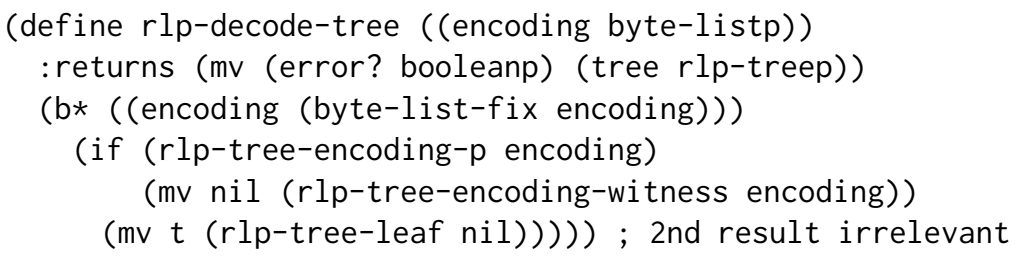

Figure 6: Declarative definition of RLP decoding in ACL2.

the theorem prohibits a valid encoding from being a strict prefix of another valid encoding.

The theorem rlp-encode-tree-unamb-prefix is proved via a case split, specified as a hint (not shown), on whether the lengths of the encodings are equal or not. If the lengths are equal, the encodings must be equal since one is a prefix of the other; this is proved automatically via library theorems about prefixp. If the lengths are not equal, a more general theorem (not shown) is used to show that the lengths must be actually equal, proving this case by contradiction. That more general theorem says that the length of an encoding is determined by the first few bytes of the encoding, because encodings start with length information. Additional hints (not shown) guide ACL2 to recognizing that those first few bytes must be the same for the two encodings if one is a prefix of the other.

\section{RLP Decoding}

\subsection{Declarative Specification}

The RLP decoding of trees from their encodings is formalized in Figure 6

The function rlp-decode-tree takes as input a list of bytes that is the purported encoding and returns as output a pair consisting of a boolean error flag and a tree. The error flag is nil exactly when the input is a valid encoding of a tree: in this case, that tree is returned, via the witness function rlp-tree-encoding-witness associated to rlp-tree-encoding-p in Figure 4 (more details on this below). Otherwise, the error flag is $t$ and the second component of the output is irrelevant.

The existentially quantified function $r l p-t r e e-e n c o d i n g-p$ is defined in terms of the witness function rlp-tree-encoding-wi tness (under the hood [17, Topic defun-sk]; see : skolem-name in Figure 4), which is axiomatized, via the matrix of the quantification, to be a right inverse of the encoding function $r l p$-encode-tree: if encoding is a valid tree encoding, then ( $r l p$-tree-encoding-witness encoding) returns tree of type rlp-treep such that (rlp-encode-tree tree) returns (mv nil encoding), i.e. no error and the original encoding. This readily implies that $r l p$-decode-tree is a right inverse of $r l p$-encode-tree, as stated by the theorem rlp-encode-tree-of-rlp-decode-tree in Figure 7; if encoding is a valid tree encoding (modulo byte-list-fix), then rlp-decode-tree succeeds and returns tree, and $r l p$-encode-tree succeeds on tree and returns the original encoding. This is proved automatically, using easily proved theorems (not shown) about $r l p$-tree-encoding-witness.

Since, as discussed in Section 3.4, rlp-encode-tree is injective, $r l p$-decode-tree is also a left inverse of $r l p$-encode-tree $4^{4}$ as stated by the theorem $r l p$-decode-tree-of-rlp-encode-tree in Figure 7; if tree is (modulo rlp-tree-fix) a tree, and rlp-encode-tree succeeds and returns encoding, then $r l p$-decode-tree succeeds on encoding and returns the original tree. This is proved via a few

\footnotetext{
${ }^{4}$ In general, if a function $f$ is injective and has a right inverse $g$, then $g$ is also a left inverse of $f$ : given the right inverse property $f(g(x))=x$, replacing $x$ with $f(y)$ yields $f(g(f(y)))=f(y)$, which the injectivity of $f$ reduces to $g(f(y))=y$, i.e. the left inverse property.
} 


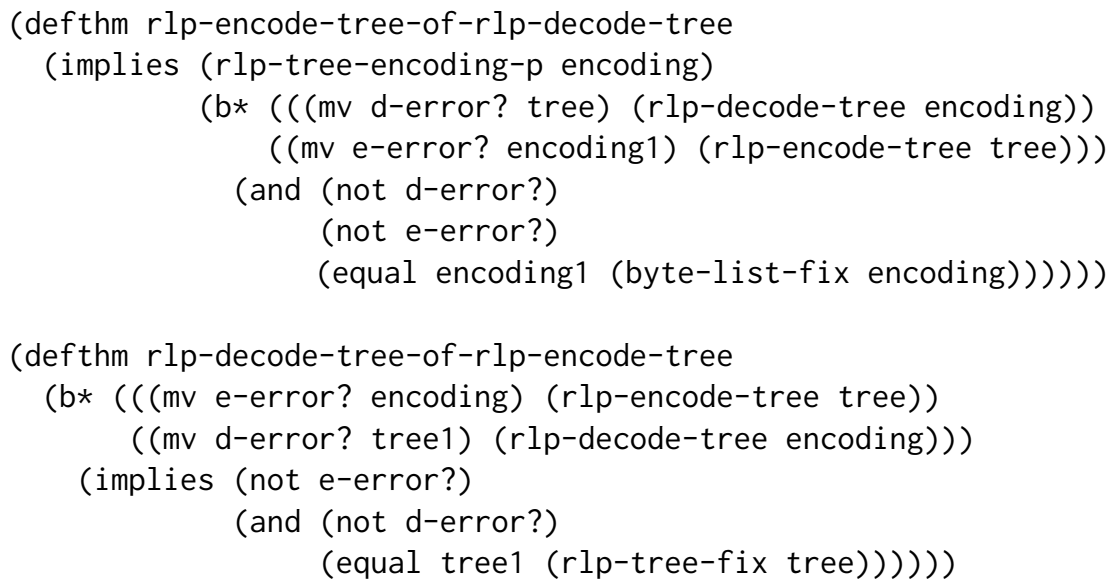

Figure 7: ACL2 theorems asserting that RLP encoding and decoding are mutual inverses.

hints (not shown) to instantiate (replacing encoding with (mv-nth 1 (rlp-encode-tree tree)) ) and use the right inverse theorem $r l p$-encode-tree-of-rlp-decode-tree, while the injectivity theorem in Figure 5 is automatically used as a rewrite rule.

Figure 6 defines the RLP decoding function as inverse of the RLP encoding function. This is a declarative, non-executable definition. YP does not explicitly define any RLP decoding function, but, clearly, it implicitly defines it as inverse of the encoding function: this implicit definition is formalized in Figure 6. WK provides Python reference code for RLP decoding, but the definition in Figure 6 is more abstract and manifestly correct.

\subsection{Executable Implementation}

The function rlp-decode-tree in Figure 6 can be regarded as a high-level specification of RLP decoding, which can be implemented by an equivalent executable function.

The executable decoding function is defined in terms of the executable RLP parser shown in Figure 8. This function is mutually recursive with the one in Figure 9. Similarly to Figure 3 , these two functions are surrounded by defines (not shown).

The function rlp-parse-tree takes as input a list of bytes and returns as output a triple consisting of an error indication (nil if parsing is successful), a decoded tree (an irrelevant one if parsing fails), and the remaining bytes after the parsed encoding ( $\mathrm{nil}$ if parsing fails 5 ). The parser stops as soon as a tree is successfully decoded (because of theorem rlp-encode-tree-unamb-prefix in Figure 5, there cannot be a longer encoding to parse), returning the remaining bytes for further parsing: thus, as lists of trees are recursively parsed, the input bytes are threaded through, and consumed chunk-wise. The function $r l p$-parse-tree-list takes as input a list of bytes and returns as output a pair consisting of an error indication (similarly to rlp-parse-tree) and a list of decoded trees; it returns no remaining bytes because it is always called (by rlp-parse-tree) on a sublist of the input of known length that must exactly encode zero or more trees. The predicate maybe-rlp-error-p recognizes nil (for no error) and error values (recognized by rlp-error-p, not shown) that convey information about different possible parsing errors; the exact errors are not shown in Figure 8 , replaced with ellipses.

\footnotetext{
${ }^{5}$ Returning nil as third result when parsing fail may seem an odd choice, compared to returning the remaining bytes whose parsing caused the error, which could convey information about the error. However, this (and more) information is already included in the error result, whose recognizer $r l p-e r r o r-p$ is mentioned a few sentences later (but not explained in detail).
} 


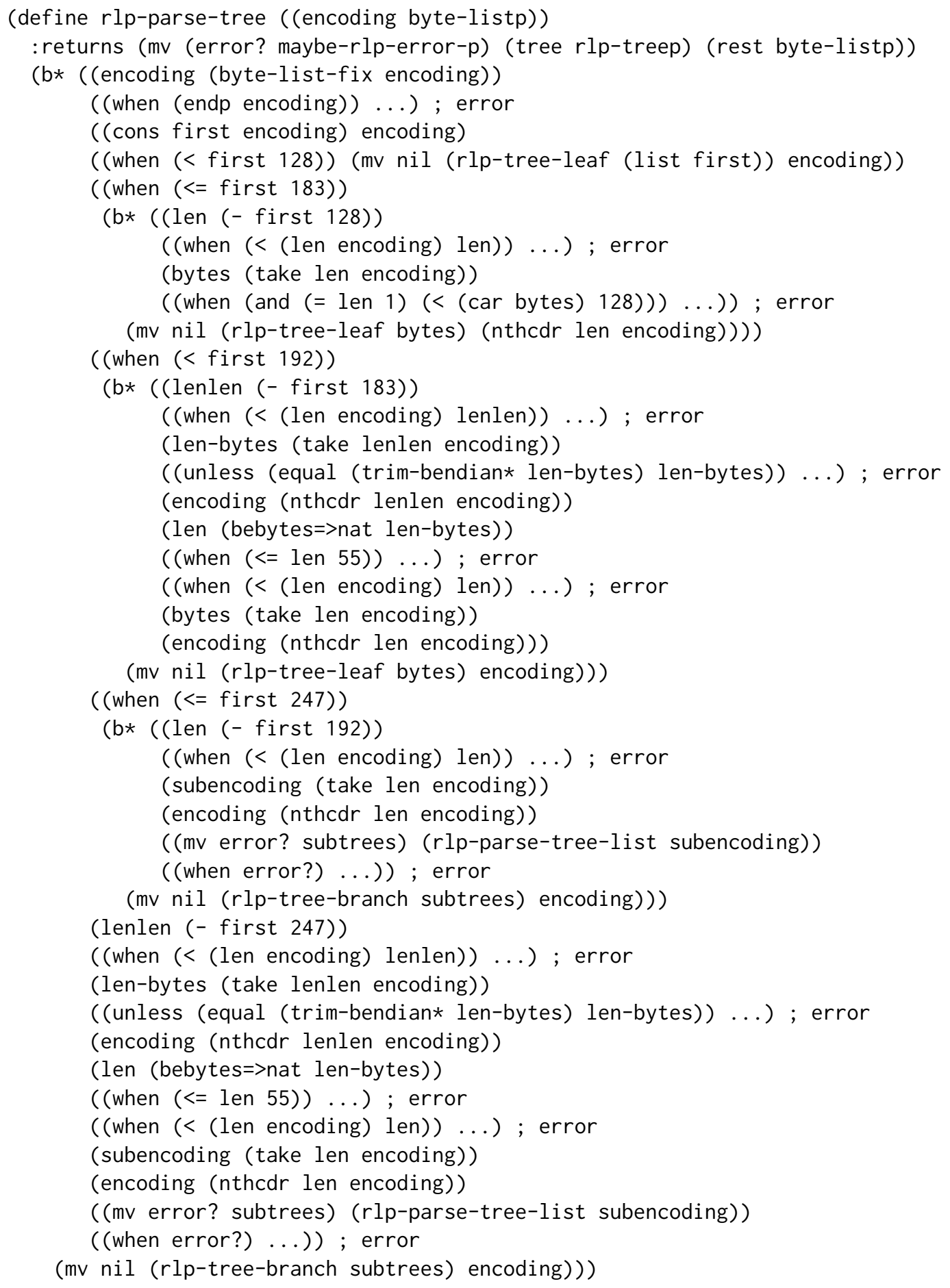

Figure 8: Executable parser of RLP encodings in ACL2 (Part 1). 


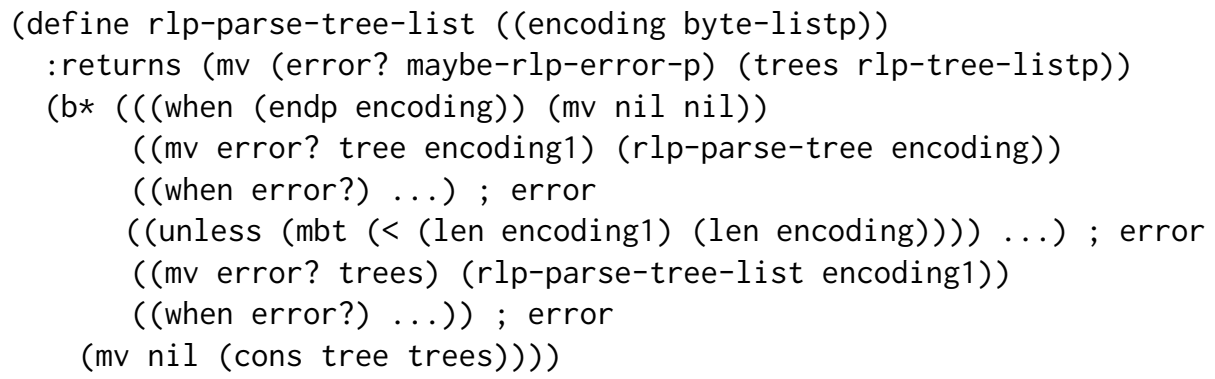

Figure 9: Executable parser of RLP encodings in ACL2 (Part 2).

The parser operates as follows (cf. the encoding functions in Figure 3):

1. It fixes the encoding input to be a list of bytes (a no-op under the guard).

2. If the input list of bytes is empty, an error is returned: RLP encodings are never empty.

3. Otherwise, the encoding input is matched to the (cons first encoding) pattern, binding the variable first to the first byte of the encoding and the (new) variable encoding to the rest of the encoding. This way, the first byte can be examined to determine what to do next.

4. If the first byte is below 128, it encodes the singleton list of itself, and so the byte is returned as a leaf tree.

5. If the first byte is in the range 128-183, the encoding must be of a byte array with length below 56 . The length is calculated: if not enough bytes occur after the first, an error is returned; otherwise, the bytes are returned as a leaf tree. An error is also returned if there is just one byte below 128; see Section 4.2.1

6. If the first byte of the encoding is in the range 184-191, the encoding must be of a byte array whose length len is encoded by the next lenlen bytes after the first one, as a base-256 big-endian noleading-zeros list. After obtaining lenlen from the first byte, an error is returned if there are not enough bytes to encode the length. An error is also returned if the encoded length has leading zeros (the library function trim-bendian* removes all the leading zeros from a list of big-endian digits); see Section 4.2.1. Otherwise, the library function bebytes=>nat, inverse of nat=>bebytes* in Figure 3, is used to calculate the length len of the encoded byte array. An error is returned if this length is below 56; see Section 4.2.1. If there are enough bytes after the first and the next lenlen bytes, they are returned as a leaf tree.

7. If the first byte of the encoding is in the range 192-247, the encoding must be of a branching tree whose subtrees have a total encoded length below 56. That exact number of bytes is passed to rlp-parse-tree-list (which is described in more detail below), which returns an error indication (nil if no error) and the list of decoded trees, which are wrapped into a branching tree and returned. As above, an error is returned if there are not enough bytes in the input. Any error from rlp-parse-tree-list is returned by rlp-parse-tree.

8. If the first byte of the encoding is in the range 248-255, the encoding must be of a branching tree whose length len is encoded by the next lenlen bytes after the first one, as a base-256 big-endian no-leading-zeros list. The processing of lenlen and len is analogous to the case in which the first byte is in the range 184-191 (explained above), including returning errors if there are leading zeros in the length or if len is below 56 (see Section 4.2.1). Similarly to the case in which the first byte is in the range 192-247, rlp-parse-tree-list is called with the exact number of bytes to parse, any error from that call is propagated, and ultimately (if no errors occur) the decoded branching tree is returned. 


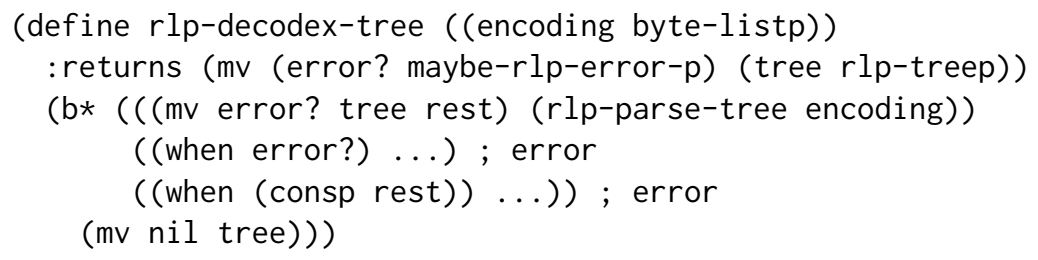

Figure 10: Executable definition of RLP decoding in ACL2.

The function rlp-parse-tree-list in Figure 9 takes as input a list of bytes purported to encode zero or more trees and returns as output a pair consisting of an error indication (nil if no error) and a list of decoded RLP trees. Unlike rlp-parse-tree, it does not return any remaining input bytes, as explained earlier.

The body of rlp-parse-tree-list decodes trees while there are input bytes available, stopping if an error occurs and propagating that error. After each tree is decoded, the remaining bytes are recursively parsed. The mbt expression serves to prove termination, as discussed below.

The termination of the mutually recursive functions $r l p$-parse-tree and $r l p$-parse-tree-list is proved automatically once a measure (not shown) is provided. The measure is lexicographic: it consists of the length of the input list of bytes, followed by a linear ordering of the two functions defined by rlp-parse-tree being smaller than rlp-parse-tree-list. When rlp-parse-tree calls rlp-parse-tree-list, the first component of the measure decreases. When rlp-parse-tree-list calls rlp-parse-tree, the first component is unchanged but the second decreases. When rlp-parsetree-list calls itself, the first component also decreases, because RLP encodings are never empty, and therefore the preceding call of $r l p$-parse-tree must have consumed some input bytes. The latter is a property of $r l p$-parse-tree, but in order to prove this property, the function must be first accepted by ACL2, requiring its termination to be proved. This circularity is broken via the run-time test (< (len encoding1) (len encoding)), which gets the function definition accepted by ACL2. The mbt around the test avoids executing the test at run time [17, Topic mbt].

The guard verification proofs, which involve the truth of the aforementioned mbt test, are automatic after proving a theorem saying that the third result of $r l p$-parse-tree, i.e. the remaining input bytes, is strictly shorter than the input. This theorem (not shown) is proved automatically after supplying a hint to expand the definition of rlp-parse-tree, which otherwise ACL2's heuristics apparently prevent from expanding.

The executable RLP decoding function for trees is defined in Figure 10; the ' $x$ ' in its name stands for 'executable', as opposed to the declaratively defined decoding function in Figure 6 . This decoding function takes as input a list of bytes, purported to be a complete encoding with no extra bytes ${ }^{6}$ and returns as output a pair consisting of an error indication (nil if decoding is successful) and a treewhose value is irrelevant if the first result is not nil. A tree is decoded by calling the parser and ensuring that there are no remaining bytes.

\subsubsection{Rejection of Invalid Quasi-Encodings}

As mentioned above, the parser rejects "quasi-encodings" of the following forms:

\footnotetext{
${ }^{6}$ This executable decoding function is meaningful as implementation of the inverse of the encoding function declaratively defined in Figure 6 It can be used when the lengths of the purported encodings are known. When the lengths are not known, e.g. when decoding from byte streams, the executable parser in Figure 8 can be used instead.
} 


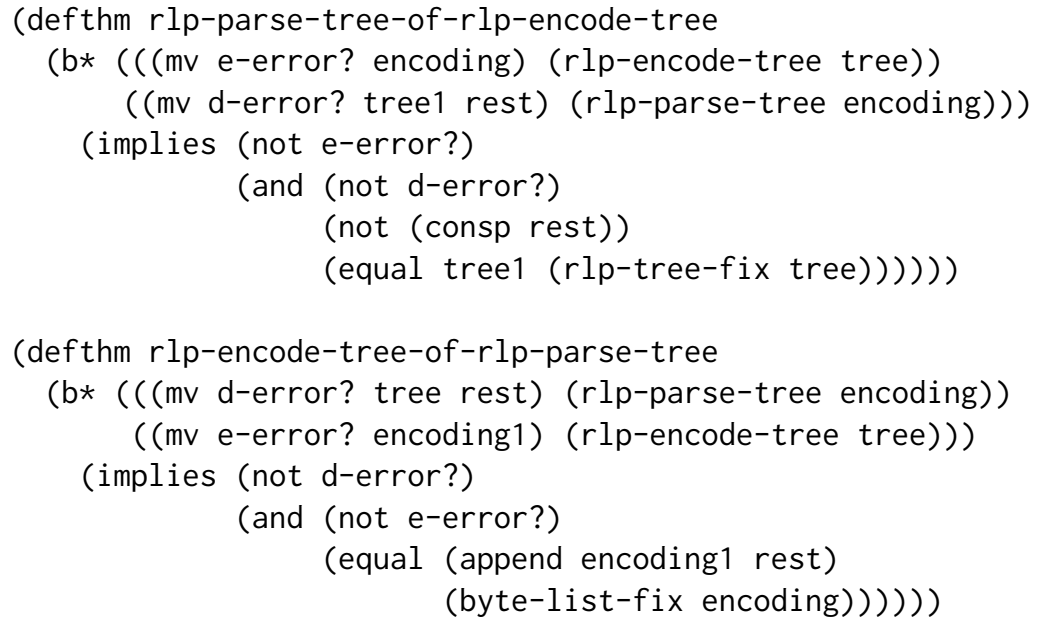

Figure 11: ACL2 theorems asserting that RLP encoding and parsing are mutual inverses.

- $[129, x]$ with $x<128$ : this "could" encode (a leaf tree consisting of) a singleton byte array $[x]$, but RLP prescribes the encoding $[x]$ in this case.

- $\left[183+l l, l_{1}, \ldots, l_{l l}, x, \ldots\right]$ with $1 \leq l l \leq 8$ and $l_{1}=0$ : this "could" encode (a leaf tree consisting of) a byte array $[x, \ldots]$ of length $l=\sum_{1 \leq i \leq l l} l_{i} \times 256^{l l-i}$, but RLP prescribes the absence of leading zeros in the base-256 big-endian representation of $l$.

- $[184, l, x, \ldots]$ with $0<l<56$ : this "could" encode (a leaf tree consisting of) a byte array $[x, \ldots]$ of length $l$, but RLP prescribes the encoding $[128+l, x, \ldots]$ in this case.

- $\left[247+l l, l_{1}, \ldots, l_{l l}, x, \ldots\right]$ with $1 \leq l l \leq 8$ and $l_{1}=0$ : this "could" encode a branching tree whose concatenated encoded subtrees have length $l=\sum_{1 \leq i \leq l l} l_{i} \times 256^{l l-i}$, but RLP prescribes the absence of leading zeros in the base-256 big-endian representation of $l$.

- $[248, l, x, \ldots]$ with $0<l<56$ : this "could" encode a branching tree whose concatenated encoded subtrees have length $l$, but RLP prescribes the encoding $[192+l, x, \ldots]$ in this case.

These are not valid encodings because they do not satisfy the predicate in Figure 4 . While they "could" be encodings in the sense mentioned above, they are "non-optimal": shorter valid encodings exist.

When implementing RLP decoding, the rejection of these quasi-encodings may be easily overlooked. My initial writing of the parser failed to reject these quasi-encodings; I discovered the problem when failing to prove the right inverse property in Section 4.3. The Python reference code in WK used to accept these quasi-encodings, and some existing RLP implementations used to accept or still accept them as well (see Section 5).

While it may seem benign to accept these quasi-encodings, they are not in the image of the RLP encoding function. Also see https://gitter.im/ethereum/research/archives/2016/07/30; search for 'consensus rule'. A possible problematic scenario is a database that uses RLP encodings as keys: if different encodings for the same item were accepted, the item could be stored multiple times in the database.

\subsection{Verification of Correctness}

The first step toward proving that the executable definition in Figure 10 is equivalent to the declarative definition in Figure 6 is to prove that the tree parsing and encoding functions are mutual inverses, which is expressed by the theorems in Figure 11 . 
The first theorem in Figure 11 says that $r l p$-parse-tree is a left inverse of $r l p$-encode-tree over the encodable trees, i.e. the parser recognizes and reconstructs all valid encodings of trees. More in detail, it says that if rlp-encode-tree succeeds, then rlp-parse-tree succeeds on the resulting encoding, returning the original tree (modulo fixing) and no remaining bytes (i.e. it consumes the whole encoding). Since rlp-parse-tree is mutually recursive with rlp-parse-tree-list, and rlp-encode-tree is mutually recursive with $r l p$-encode-tree-list, that theorem is proved at the same time as another theorem (not shown) that says that $r l p$-parse-tree-list is a left inverse of $r l p$-encode-tree-list. These two theorems are proved by induction on rlp-encode-tree and rlp-encode-tree-list, via the make-flag macro [17, Topic make-flag]. The proof is automatic once the definitions of these and other functions are enabled, and some hints (not shown) are provided to force the expansion of some calls of rlp-parse-tree; see the documentation [17, Topic $r l p]$ for details. The proof also makes use of a previously proved more general rewrite rule (not shown) saying that if $r l p$-parse-tree succeeds on encoding, returning a tree and some remaining bytes rest, it also succeeds on an extended input (append encoding more-bytes), returning the same tree and (append rest more-bytes) as remaining bytes.

The second theorem in Figure 11 says that rlp-parse-tree is a right inverse of rlp-encode-tree over the valid tree encodings, i.e. the parser recognizes and reconstructs only valid encodings of trees: if it accepted an invalid encoding and returned a tree, $r l p$-encode-tree would have to map that tree back to the encoding, which would be therefore valid, contradicting the hypothesis that it is invalid. More in detail, the theorem says that if rlp-parse-tree succeeds, then rlp-encode-tree succeeds on the resulting tree, returning the prefix of the original encoding (modulo fixing) that omits the remaining bytes returned by rlp-parse-tree. Analogously to the left inverse theorem described above, this theorem is proved at the same time as another theorem (not shown) about rlp-parse-tree-list and rlp-encode-tree-list, via make-flag; the proof, by induction on rlp-parse-tree and rlp-parsetree-list 7 , is automatic once the definitions of these and other functions are enabled.

If the parser accepted the quasi-encodings discussed in Section 4.2.1, the left inverse theorem would still hold, but the right inverse theorem would not.

The fact that $r l p$-decodex-tree in Figure 10 is both a left and a right inverse of $r l p$-encode-tree easily follows from the theorems in Figure 11. This fact is asserted by two theorems (not shown) that are written as in Figure 7 but where rlp-decode-tree is replaced with rlp-decodex-tree. The left inverse theorem is proved automatically once the definition of $r l p$-decodex-tree is enabled; the first theorem in Figure 11 applies as a rewrite rule. The right inverse theorem, besides enabling the definition of rlp-decodex-tree, requires a couple of hints (not shown) to use the second theorem in Figure 11 . since the append in it does not make it readily applicable as a rewrite rule in this case.

The equivalence of $r l p$-decodex-tree and $r l p$-decode-tree is stated by the theorem in Figure 12 Since $r l p$-decode-tree returns a boolean error result while $r l p$-decodex-tree returns a richer range of error results, the first results of these two functions are only iff-equivalent, i.e. one is nil if and only if the other one is nil; their second results are always equal instead.

The theorem in Figure 12 is proved by cases on whether ( $r$ lp-tree-encoding-p encoding) holds or not. A preliminary lemma (not shown) is proved, with a few hints and a couple of simple intermediate lemmas, asserting the equivalence of (i) $r l p$-tree-encoding-p returning $t$ and (ii) $r l p$-decodex-tree returning a nil error result. If $r l p$-tree-encoding-p holds:

\footnotetext{
${ }^{7}$ In ACL2, as in NQHTM [4, Chapt. 15], induction is applicable when the arguments that decrease in the recursion are variables. Thus, while the left inverse theorems are proved by induction on rlp-encode-tree and rlp-encode-tree-list, the right inverse theorems are proved by induction on $r l p$-parse-tree and $r l p$-parse-tree-list.
} 


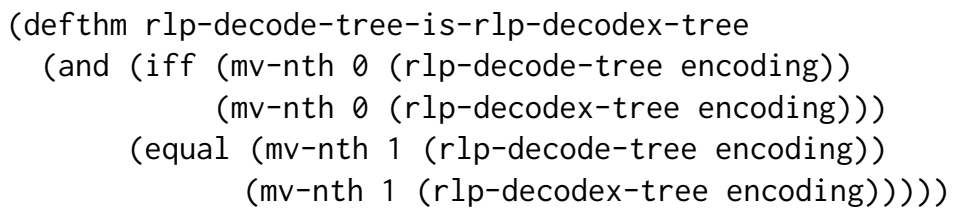

Figure 12: ACL2 theorem asserting the correctness of the executable RLP decoder.

- The first conjunct in Figure 12 is proved via a few hints, using the aforementioned lemma and the definition of rlp-decode.

- The second conjunct in Figure 12 is proved via a few hints, from rlp-encode-tree-injective and the right inverse properties of $r l p$-decode-tree and rlp-decodex-tree. 8

If $r l p$-tree-encoding-p does not hold, both conjuncts are proved via a few hints, using the aforementioned preliminary lemma and the definitions of $r l p$-decode-tree and $r l p$-decodex-tree.

\section{Related Work and Impact}

RLP is formally defined in YP.9 ${ }^{9}$ Based on the ACL2 development, I contributed some improvements to that definition (see Pull Requests 700, 736, 739, 742, 745, and 746 at https://gi thub. com/ethereum/ yellowpaper) and helped close some outstanding items (see Pull Request 648 and Issue 116 at https: //github.com/ethereum/yellowpaper).

RLP is informally defined in WK. Previously, the Python reference code for RLP decoding in WK accepted the quasi-encodings described in Section 4.2.1. Based on the ACL2 development, I contributed a fix to reject those quasi-encodings (see Issue 688 at https://github.com/ethereum/wiki).

The KEVM [7] [22] includes executable specifications of RLP encoding and decoding ${ }^{10}$ The decoding specification covers all encodings, while the encoding specification only covers byte arrays and some data types that are encoded like byte arrays. There are no theorems stating that the specified encoding and decoding are mutual inverses. The decoding specification appears to accept some of the quasi-encodings described in Section 4.2.1 (see Issue 413 at https://gi thub.com/kframework/evm-semantics).

The Lem EVM [9] [8] includes a partial (apparently in progress) Isabelle/HOL specification of RLP encoding and decoding ${ }^{11}$ The type of RLP trees is much like Figure 2. There is a complete specification of RLP encoding, but only a partial specification of RLP decoding. There are no theorems stating that the specified encoding and decoding are mutual inverses.

There are several implementations of RLP, in libraries and Ethereum clients, written in mainstream programming languages. Some of these implementations used to accept, or still accept, the quasiencodings described in Section 4.2.1 (e.g. see Issue 1639 at https://github.com/ethereum/aleth and Issue 49 at https://gi thub.com/paritytech/parity-common).

The Ethereum test suite [19] contains JSON-formatted tests for all Ethereum implementations, including tests for RLP encoding and decoding. Previously, this test suite included a few tests for rejecting some, but not all, of the five kinds of quasi-encodings described in Section 4.2.1. Based on the ACL2 development, I contributed additional tests to cover all the five kinds of quasi-encodings (see Pull Request

\footnotetext{
${ }^{8}$ In general, if an injective function $f$ has right inverses $g$ and $h$, then $g=h$ : from the right inverse properties $f(g(x))=x=$ $f(h(x))$, injectivity gives $g(x)=h(x)$.

${ }^{9}$ Using "pencil-and-paper" mathematical notation, not in a theorem prover or similar tool.

${ }^{10}$ The KEVM is an evolving artifact. The following assertions are current at the time of this writing.

${ }^{11}$ The Lem EVM is an evolving artifact. The following assertions are current at the time of this writing.
} 
612 at https: //github.com/ethereum/tests).

There is extensive work on formal verification and analysis of (Ethereum and other) smart contracts, for which just some references are provided here [1] [2] [3] [5] [6] [7] [9] [10] [13] [14] [15] [16]. That work is complementary to the ACL2 RLP development, whose focus is (a component of) the platform that runs smart contracts. However, as mentioned in Section 1, this platform-focused work can support smart contract verification, by providing the underlying formal semantics of smart contracts.

\section{Future Work}

Alternative, perhaps more efficient, implementations of the RLP parser and decoder in Section 4.2 could be written and verified, comparing their proof efforts and techniques with Section 4.3 .

It could be investigated how to derive, via stepwise refinement, verified and efficient RLP parser and decoder implementations from the declarative specification in Section 4.1, using the APT (Automated Program Transformations) toolkit [17, Topic APT]. This may require the development of additional, but more generally applicable, APT transformation tools.

The RLP parser and decoder in Section 4.2 are given the complete purported encodings, or more, as input. However, practical implementations may read the input bytes as needed, e.g. from a socket. It could be investigated how to extend the specification, implementation, and proof to accommodate this approach. In this case, additional customizable length checks should be probably added, to thwart denialof-service-style attacks consisting in supplying the first few bytes of very long encodings, e.g. close to the $2^{64}$ limits.

The RLP parser and decoder in Section 4.2 process encodings completely, i.e. to the full depth of the RLP trees. However, practical implementations may process encodings up to a specified tree depth initially, then to lower depths as needed. It could be investigated how to extend the specification, implementation, and proof to accommodate this approach. These new parser and decoder will need to accept invalid encodings that are however valid up to the specified depths, requiring a corresponding weakening of their specification.

While RLP is an important component of Ethereum, clearly a lot more work remains to extend the development described in this paper to a complete Ethereum client. This remaining work is in progress [18, Path books/kestrel/ethereum] [17, Topic ethereum].

\section{Acknowledgments}

- The Ethereum Foundation for supporting this work.

- Eric McCarthy for useful discussions on RLP and for valuable comments on the initial draft of this paper, including suggesting the RLP database scenario mentioned at the end of Section 4.2.1.

- The anonymous reviewers for useful suggestions.

\section{References}

[1] Sidney Amani, Myriam Bégel, Maksym Bortin \& Mark Staples (2018): Towards Verifying Ethereum Smart Contract Bytecode in Isabelle/HOL. In: Proc. 7th International Conference on Certified Programs and Proofs (CPP), doi $10.1145 / 3167084$

[2] Danil Annenkov \& Bas Spitters (2019): Towards a Smart Contract Verification Framework in Coq. In: Proc. 1st Workshop on Formal Methods for Blockchains (FMBC). 
[3] Karthukeyan Bhargavan, Antoine Delignat-Lavaud, Cédric Fournet, Anitha Gollamudi, Georges Gonthier, Nadim Kobeissi, Natalia Kulatova, Aseem Rastogi, Thomas Sibut-Pinote, Nikhil Swamy \& Santiago ZanellaBéguelin (2016): Formal Verification of Smart Contracts. In: Proc. 16th ACM SIGSAC Workshop on Programming Languages and Analysis for Security (PLAS), pp. 91-96, doi $10.1145 / 2993600.2993611$.

[4] Robert S. Boyer \& J Strother Moore (1979): A Computational Logic. Academic Press, doi:10.1016/C20130-10411-4

[5] Ting Chen, Xiaoqi Li, Xiapu Luo \& Xiaosong Zhang (2017): Under-Optimized Smart Contracts Devour Your Money. In: Proc. 24th IEEE International Conference on Software Analysis, Evolution and Reengineering (SANER), pp. 442-446, doi 10.1109/SANER.2017.7884650

[6] Sylvain Conchon, Alexandrina Korneva \& Fatiha Zaïdi (2019): Verifying Smart Contracts with Cubicle. In: Proc. 1st Workshop on Formal Methods for Blockchains (FMBC).

[7] Everett Hildenbrandt, Manasvi Saxena, Xiaoran Zhu, Nishant Rodrigues, Philip Daian, Dwight Guth \& Grigore Roşu (2017): KEVM: A Complete Semantics of the Ethereum Virtual Machine. Technical Report, University of Illinois Urbana-Champaign. http://hdl. handle.net/2142/97207

[8] Yoichi Hirai: Formalization of Ethereum Virtual Machine in Lem. https://github.com/pirapira/ eth-isabelle

[9] Yoichi Hirai (2017): Defining the Ethereum Virtual Machine for Interactive Theorem Provers. In: Proc. 1st Workshop on Trusted Smart Contracts (WTSC), LNCS, doi:10.1007/978-3-319-70278-0_33.

[10] Sukrit Kalra, Seep Goel, Mohan Dhawan \& Subodh Sharma (2018): ZEUS: Analyzing Safety of Smart Contracts. In: Proc. 25th Annual Network and Distributed System Security Symposium (NDSS), doi $10.14722 /$ ndss.2018.23082.

[11] Matt Kaufmann \& J Strother Moore: The ACL2 Theorem Prover: Web Site. http://www.cs.utexas.edu/ users/moore/acl2

[12] Kestrel Institute: ACL2 Ethereum Project. https://www.kestrel.edu/home/projects/ethereum/

[13] Loi Luu, Duc-Hiep Chu, Hrishi Olickel, Prateek Saxena \& Aquinas Hobor (2016): Making Smart Contracts Smarter. In: Proc. 23rd Conference on Computer and Communication Security (CCS), pp. 254-269, doi $10.1145 / 2976749.2978309$

[14] Matteo Marescotti, Martin Blicha, Antti E. J. Hyvärinen, Sepideh Asadi \& Natasha Sharygina (2018): Computing Exact Worst-Case Gas Consumption for Smart Contracts. In: Proc. 8th International Symposium on Leveraging Applications of Formal Methods (ISoLA), LNCS 11247, pp. 450-465, doi:10.1007/978-3-03003427-6_33.

[15] Zeinab Nehaï \& François Bobot (2019): Deductive Proof of Industrial Smart Contracts Using Why3. In: Proc. 1st Workshop on Formal Methods for Blockchains (FMBC).

[16] Ivica Nikolić, Aashish Kolluri, Ilya Sergey, Prateek Saxena \& Aquinas Hobor (2018): Finding the Greedy, Prodigal, and Suicidal Contracts at Scale. In: Proc. 34th Annual Computer Security Applications Conference (ACSAC), pp. 653-663.

[17] The ACL2 Community: The ACL2 Theorem Prover and Community Books: Documentation. http://www. cs.utexas.edu/ moore/acl2/manuals/current/manual.

[18] The ACL2 Community: The ACL2 Theorem Prover and Community Books: Source Code. http://gi thub. com/acl2/acl2.

[19] The Ethereum Community: The Ethereum Test Suite. https://github.com/ethereum/tests

[20] The Ethereum Community: The Ethereum Web Site. https://ethereum.org.

[21] The Ethereum Community: The Ethereum Wiki. https://github.com/ethereum/wiki

[22] The KEVM Team: JelloPaper: Human Readable Semantics of EVM in K. https://jellopaper .org.

[23] Gavin Wood: Ethereum: A Secure Decentralized Generalised Transaction Ledger. https://github.com/ ethereum/yellowpaper 